\title{
Immunogenicity and Optimal Timing of 13-Valent Pneumococcal Conjugate Vaccination during Adjuvant Chemotherapy in Gastric and Colorectal Cancer: A Randomized Controlled Trial
}

\author{
Wonyoung Choi, $\mathrm{MD}, \mathrm{PhD}^{1}$ \\ Jong Gwang Kim, MD, PhD² \\ Seung-Hoon Beom, MD, PhD \\ Jun-Eul Hwang, MD, PhD' \\ Hyun-Jung Shim, MD, PhD ${ }^{1}$ \\ Sang-Hee Cho, MD, PhD 1 \\ Min-Ho Shin, MD, PhD ${ }^{4}$ \\ Sin-Ho Jung, $P h D^{5}$ \\ Ik-Joo Chung, MD, PhD ${ }^{1}$ \\ Joon Young Song, MD, $\mathrm{PhD}^{6}$ \\ Woo Kyun Bae, MD, PhD ${ }^{1}$
}

*A list of author's affiliations appears at the end of the paper.

Correspondence: Woo Kyun Bae, MD, PhD

Division of Hematology-Oncology,

Department of Internal Medicine, Chonnam

National University Hwasun Hospital,

322 Seoyang-ro, Hwasun 58128, Korea

Tel: 82-61-379-7623

Fax: 82-61-379-8009

E-mail: drwookyun@jnu.ac.kr

Co-correspondence: Joon Young Song, MD, PhD Division of Infectious Disease, Department of Internal Medicine, Korea University Guro Hospital, Korea University College of Medicine, 148 Gurodong-ro, Guro-gu, Seoul 08308, Korea

Tel: 82-2-2626-3052

Fax: 82-2-2626-1105

E-mail: infection@korea.ac.kr

Received April 8, 2019

Accepted July 7, 2019

Published Online July 9, 2019

\begin{abstract}
Purpose
Pneumococcal vaccination (13-valent pneumococcal conjugate vaccine [PCV13]) is recommended to cancer patients undergoing systemic chemotherapy. However, the optimal time interval between vaccine administration and initiation of chemotherapy has been little studied in adult patients with solid malignancies.
\end{abstract}

\section{Materials and Methods}

We conducted a prospective randomized controlled trial to evaluate whether administering PCV13 on the first day of chemotherapy is non-inferior to vaccinating 2 weeks prior to chemotherapy initiation. Patients were randomly assigned to two study arms, and serum samples were collected at baseline and 4 weeks after vaccination to analyze the serologic response against Streptococcus pneumoniae using a multiplexed opsonophagocytic killing assay.

\section{Results}

Of the 92 patients who underwent randomization, 43 patients in arm A (vaccination 2 weeks before chemotherapy) and 44 patients in arm $B$ (vaccination on the first day of chemotherapy) were analyzed. Immunogenicity was assessed by geometric mean and fold-increase of post-vaccination titers, seroprotection rates (percentage of patients with post-vaccination titers $>1: 64$ ), and seroconversion rates (percentage of patients with $>4$-fold increase in post-vaccination titers). Serologic responses to PCV13 did not differ significantly between the two study arms according to all three types of assessments.

\section{Conclusion}

The overall antibody response to PCV13 is adequate in patients with gastric and colorectal cancer during adjuvant chemotherapy, and no significant difference was found when patients were vaccinated two weeks before or on the day of chemotherapy initiation.

\section{Introduction}

Pneumococcal vaccination is recommended for cancer patients who undergo systemic chemotherapy due to the possibility of immunosuppression and consequent risk of invasive pneumococcal disease. The latest guideline from the
Key words

Chemotherapy, Vaccination, Stomach neoplasms, Colorectal neoplasms
Infectious Diseases Society of America (IDSA) states that patients in this group should receive the sequential 13-valent pneumococcal conjugate vaccine (PCV13) and 23-valent pneumococcal polysaccharide vaccine (PPSV23) immunization [1]. The guideline also states that the timing of vaccination should be at least 2 weeks prior to starting chemotherapy, and that vaccine administered during chemotherapy 
should not be considered valid. Despite their strong level of recommendation, the quality of evidence is low because there have not been sufficient clinical studies focusing on the immunogenicity and optimal timing of PCV13 administration in cancer patients undergoing systemic chemotherapy. Furthermore, recent chemotherapy in solid tumors has rapidly changed to less toxic treatments in an outpatient setting. Therefore, the guidelines concerning when this vaccine should be administered should be changed.

The immunogenicity of pneumococcal vaccines in cancer patients was shown in previous studies performed with PPSV23, which demonstrated that adequate immune responses could be induced in patients undergoing chemotherapy $[2,3]$. As for PCV13, a recent study reported its safety and immunogenicity in children who completed cancer treatment [4]. However, data on PCV13 in adult cancer patients are lacking.

There is insufficient evidence to conclude an optimal time interval between vaccine administration and chemotherapy. However, a recent study performed with influenza vaccines in cancer patients reported that the overall antibody response did not differ significantly when vaccination was performed early (day 5) or late (day 16) during a 3-week chemotherapy cycle in breast and colon cancer patients [5]. This study implied that a shorter interval between vaccination and chemotherapy could result in comparable immune responses.

In light of these previous studies, we hypothesized that administering PCV13 on the first day of chemotherapy would be as effective as vaccinating 2 weeks prior to treatment initiation. As gastric and colorectal cancers are prevalent in Korea and share similar chemotherapeutic adjuvant therapy regimens, we designed a non-inferiority trial in these patients to validate our hypothesis.

\section{Materials and Methods}

\section{Study design}

This study was a prospective randomized controlled noninferiority trial conducted in three cancer centers in Korea from March 2016 to March 2018. The primary study objective was to evaluate whether the immunogenicity of PCV13 given on the first day of adjuvant chemotherapy is non-inferior to vaccinating 2 weeks prior to chemotherapy initiation. Patients were randomly assigned to receive PCV13 either 2 weeks before (day -14) or on the day of (day 0 ) the start of adjuvant chemotherapy by block randomization. Venous blood samples $(10 \mathrm{~mL})$ were collected on day 0 and on postvaccination day $28 \pm 7$ to assess the immunogenicity of PCV13.

\section{Study subjects}

Study subjects (aged $\geq 19$ years) were deemed eligible if they had undergone surgical resection for primary gastric or colorectal cancer, were scheduled for adjuvant chemotherapy (fluoropyrimidine \pm oxaliplatin), had an Eastern Cooperative Oncology Group performance status $\leq 2$, and showed adequate hematological, renal, and hepatic function within 2 weeks of randomization using the following criteria: absolute neutrophil count $\geq 1,500 / \mathrm{mm}^{3}$, platelet count $\geq$ $100,000 / \mathrm{mm}^{3}$, serum creatinine $\leq 1.5$-fold the upper limit of normal (ULN) or estimated glomerular filtration rate $>50$ $\mathrm{mL} / \mathrm{min}$ calculated using the Cockcroft-Gault equation, serum aspartate aminotransferase and alanine aminotransferase $\leq 3$-fold the ULN, and total bilirubin $\leq 2$-fold the ULN. Exclusion criteria included previous pneumococcal vaccination, hypersensitivity to diphtheria toxin or other compounds contained in the vaccine, history of immunodeficiency disorders or autoimmune diseases, use of immunomodulatory drugs within 3 months of randomization, and signs of infection within 24 hours of vaccination.

\section{Vaccine}

The PCV13 (Prevenar-13, Wyeth Pharmaceuticals, Madison, NJ) contains capsular antigens of serotypes 1, 3, 4, 5, 6A, 6B, 7F, 9V, 14, 18C, 19A, 19F, and 23F of Streptococcus pneumoniae, conjugated to diphtheria $\mathrm{CRM}_{197}$ protein, and is supplied in a $0.5-\mathrm{mL}$ prefilled syringe. The vaccine was administered by intramuscular injection in the deltoid area of each patient.

\section{Primary endpoint and sample size estimation}

The primary endpoint was serotype-specific seroprotection after PCV13 administration. Based on historical data, the seroprotection rate of PCV13 was assumed to be $85 \%$ [6,7]. If the non-inferiority margin was set to $20 \%$, then a sample size of 41 patients in each arm would provide a power of $90 \%$ with a two-sided alpha level of 0.05 using Fisher exact test to demonstrate non-inferiority of arm $B$ (vaccination on the first day of chemotherapy) to arm A (vaccination 2 weeks before initiating chemotherapy). Assuming a dropout rate of approximately 10\%, 46 patients were enrolled in each arm.

\section{Immunogenicity assessment}

Multiplex opsonophagocytic killing assays (MOPAs) for serotypes 5, 6B, 18C, and 19A were performed on collected serum samples. Four serotypes were selected based on the nasopharyngeal carriage rate and invasive disease potential according to the serotype-specific capsular structure and 


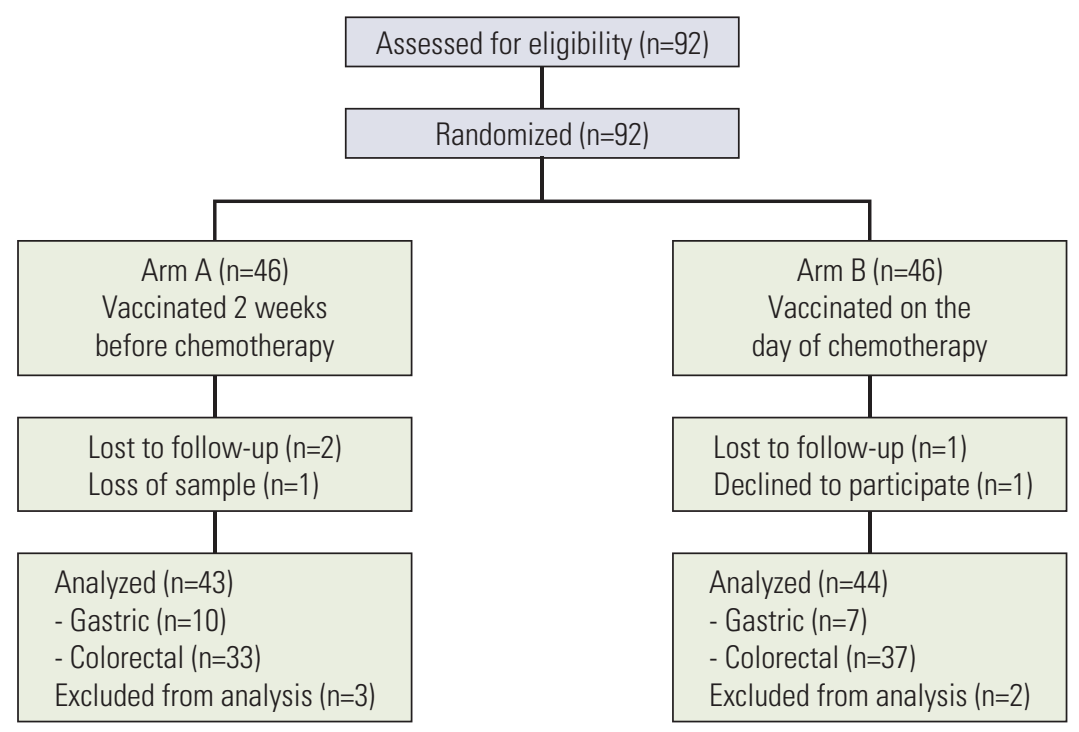

Fig. 1. Study diagram.

thickness, which might also affect the immunogenicity [8]. The titer of opsonophagocytic activity (OPA) was defined as the serum dilution ratio that killed $50 \%$ of bacteria, as previously described $[9,10]$. A detailed protocol is posted online at http://www.vaccine.uab.edu. The immunogenicity in the two study arms for each serotype was compared using the following three assessments: geometric mean and foldincrease of post-vaccination titers, percentage of patients with post-vaccination OPA titers $>1: 64$ (seroprotection rate), and percentage of patients with $>4$-fold increase in post-vaccination OPA titers (seroconversion rate).

\section{Statistical analysis}

All statistical analyses were performed using SPSS for Windows ver. 20.0 (IBM Corp., Armonk, NY). Descriptive statistics were reported as numbers and percentages of patients. OPA titers were expressed as geometric means with 95\% confidence intervals (CIs). Geometric mean titers (GMTs) and their mean fold increases were analyzed using Student's $\mathrm{t}$ test. Categorical variables were analyzed using the chisquared test (Fisher exact test). A two-sided $\mathrm{p}$-value $<0.05$ was considered to indicate statistical significance.

\section{Ethical statement}

This study was approved by the institutional review board of Chonnam National University Hwasun Hospital (IRB No. CNUHH-2015-126) and registered at the Clinical Research Information Service (registration No. КСТ0003379). This study was conducted in concordance with the Declaration of Helsinki and Good Clinical Practice. All patients provided written informed consent.

\section{Results}

\section{Baseline characteristics}

In total, 92 patients were assessed for eligibility and randomly assigned to either arm A, with PCV13 administration 2 weeks before starting adjuvant chemotherapy, or arm B, with PCV13 administration on the first day of adjuvant chemotherapy. Eighty-seven patients ( $\operatorname{arm~A,~n=43;~} \operatorname{arm~B,~n=44)~}$ were included in the final assessment of immunogenicity (Fig. 1). Baseline characteristics including sex, age, type of cancer, and chemotherapeutic regimen were indistinguishable between the two arms (Table 1).

\section{Immunogenicity assessed by geometric mean titers of OPA}

The geometric mean post-vaccination titer (GMT) of OPA with $95 \%$ CIs has been suggested to be an appropriate parameter for antibody response, accounting for its skewed distribution [11,12]. The GMT levels varied by serotype, with serotypes $6 \mathrm{~B}$ and $19 \mathrm{~A}$ showing higher titers than serotype 5 . Nevertheless, GMTs were consistently increased for all four 
Table 1. Baseline characteristics of gastric and colorectal cancer patients

\begin{tabular}{|c|c|c|c|}
\hline Characteristic & Arm A & Arm B & p-value \\
\hline No. of patients & 43 & 44 & \\
\hline \multicolumn{4}{|l|}{ Sex } \\
\hline Male & $24(55.8)$ & $28(63.6)$ & 0.516 \\
\hline Female & $19(44.2)$ & $16(36.4)$ & \\
\hline Age, mean (range, yr) & $59.9(42.0-77.6)$ & $59.1(29.1-78.9)$ & 0.678 \\
\hline \multicolumn{4}{|l|}{ Type of cancer } \\
\hline Gastric cancer & $10(23.3)$ & $7(15.9)$ & 0.429 \\
\hline Colorectal cancer & $33(76.7)$ & $37(84.1)$ & \\
\hline \multicolumn{4}{|l|}{ Chemotherapeutic regimen } \\
\hline Fluoropyrimidine $e^{\mathrm{a})}$ & $16(37.2)$ & $9(20.5)$ & 0.101 \\
\hline 5-FU/ leucovorin & 2 & 2 & \\
\hline Capecitabine & 10 & 6 & \\
\hline S-1 & 4 & 1 & \\
\hline Fluoropyrimidine+Oxaliplatin ${ }^{\text {b) }}$ & $27(62.8)$ & $35(79.5)$ & \\
\hline FOLFOX & 10 & 14 & \\
\hline CAPOX & 17 & 21 & \\
\hline
\end{tabular}

Values are presented as number $(\%)$ unless otherwise indicated. ${ }^{a}$ Fluoropyrimidine regimens include 5-fluorouracil/leucovorin, capecitabine, or S-1, b)Fluoropyrimidine+oxaliplatin regimens include FOLFOX or CAPOX.

Table 2. GMTs of opsonophagocytic activity

\begin{tabular}{|c|c|c|c|c|c|c|c|}
\hline \multirow{2}{*}{ Serotype } & \multirow{2}{*}{ Arm } & \multicolumn{3}{|c|}{ Pre-vaccination } & \multicolumn{3}{|c|}{ Post-vaccination } \\
\hline & & GMT & $95 \% \mathrm{CI}$ & p-value & GMT & $95 \% \mathrm{CI}$ & p-value \\
\hline \multirow[t]{2}{*}{5} & A & 5 & 3-7 & 0.928 & 338 & $167-693$ & 0.712 \\
\hline & B & 5 & $3-7$ & & 405 & $210-741$ & \\
\hline \multirow[t]{2}{*}{$6 \mathrm{~B}$} & A & 521 & $185-1,288$ & 0.958 & 8,913 & $5,858-12,966$ & 0.712 \\
\hline & B & 505 & $252-1,022$ & & 10,495 & 7,920-13,341 & \\
\hline \multirow[t]{2}{*}{$18 \mathrm{C}$} & A & 100 & 53-195 & 0.935 & 4,280 & $2,659-6,631$ & 0.673 \\
\hline & B & 104 & $52-798$ & & 4,955 & $2,760-7,943$ & \\
\hline \multirow[t]{2}{*}{$19 \mathrm{~A}$} & A & 436 & $224-824$ & 0.439 & 7,586 & $4,365-11,382$ & 0.766 \\
\hline & B & 308 & $176-529$ & & 6,947 & $4,879-9,456$ & \\
\hline
\end{tabular}

Pre- and post-vaccination geometric mean titers (GMTs) of opsonophagocytic activity with 95\% confidence intervals (CIs) in the two study arms for serotypes 5, 6B, 18C, and 19A.

serotypes after vaccination and did not differ significantly between the two study arms for the pre- or post-vaccination states (Table 2). The GMT-fold increase was higher for serotype 5 and lower for serotypes 6B and 19A but did not show any significant difference when compared between the two study arms (Table 3).

In subgroup analyses, the GMT results and mean fold increases in OPA titers were stratified by cancer type (gastric cancer vs. colorectal cancer) and chemotherapeutic regimen (fluoropyrimidine vs. fluoropyrimidine+oxaliplatin). When stratified by cancer type, pre- and post-vaccination GMTs did not differ significantly among all four serotypes between the two study arms for the two types of cancers (S1 Table). However, when stratified by chemotherapeutic regimen, there were some differences in the post-vaccination GMTs. In the fluoropyrimidine-treated group, post-vaccination GMTs were higher in arm A for serotypes 6B $(p=0.048)$ and 19A $(p=0.015)$. In the fluoropyrimidine+oxaliplatin-treated group, arm $B$ showed higher post-vaccination GMTs for serotype $6 \mathrm{~B}$ $(p=0.030)$ (S2 Table). However, geometric means of fold increases in OPA titers were comparable between the two study arms for all four serotypes when stratified by cancer 
Table 3. Fold increase of post-vaccination geometric mean titers

\begin{tabular}{|c|c|c|c|c|c|}
\hline \multirow[b]{2}{*}{ Serotype } & \multicolumn{2}{|c|}{ Arm A $(n=43)$} & \multicolumn{2}{|c|}{ Arm B (n=44) } & \multirow[b]{2}{*}{ p-value } \\
\hline & $\begin{array}{l}\text { Geometric mean } \\
\text { fold increase }\end{array}$ & $95 \% \mathrm{CI}$ & $\begin{array}{l}\text { Geometric mean } \\
\text { fold increase }\end{array}$ & $95 \% \mathrm{CI}$ & \\
\hline 5 & 72.15 & $35.93-144.86$ & 88.60 & $47.55-165.06$ & 0.658 \\
\hline $6 \mathrm{~B}$ & 17.09 & $6.83-42.76$ & 20.81 & 9.94-43.55 & 0.737 \\
\hline $18 \mathrm{C}$ & 42.73 & $19.34-94.41$ & 47.60 & 24.53-92.37 & 0.833 \\
\hline $19 \mathrm{~A}$ & 17.41 & 9.93-30.52 & 22.54 & $12.56-40.46$ & 0.522 \\
\hline
\end{tabular}

Fold increase in geometric mean titers after vaccination in the two study arms for serotypes 5, 6B, 18C, and 19A. CI, confidence interval.
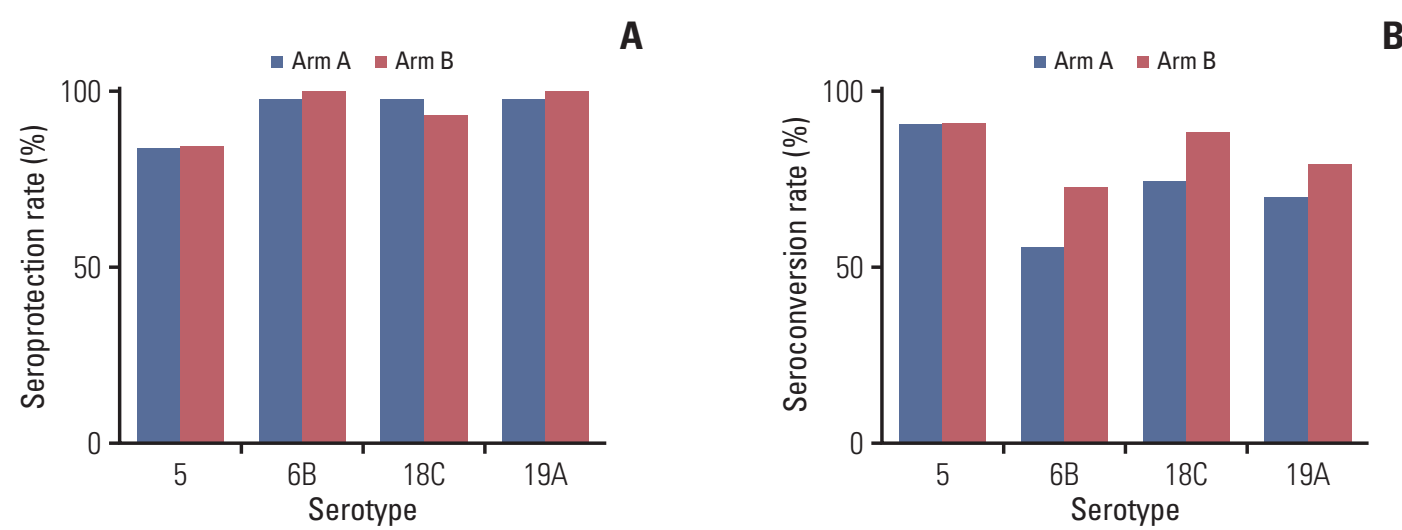

Fig. 2. Seroprotection and seroconversion rates between the two study arms. Bar plots for the seroprotection (A) and seroconversion (B) rates of the two study arms for serotypes 5, 6B, 18C, and 19A.

type or chemotherapeutic regimen (S3 and S4 Tables).

\section{Immunogenicity assessed by seroprotection rate}

Antibody levels above a certain threshold should provide adequate protection towards targeted strains. Therefore, the proportion of patients who actually reach this state of protection, known as the seroprotection rate, may be interpreted as a clinical parameter of immunogenicity. An OPA titer of 1:64 has been suggested as a threshold for evaluating an adequate response to pneumococcal vaccination [13]. Thus, the percentages of patients with OPA titers $>64$ for each serotype were compared. Seroprotection rates were generally high for all four serotypes, with percentages $>80 \%$ for serotype 5 , and $>90 \%$ for the other three serotypes. No significant intergroup difference was observed (Fig. 2A, S5 Table).

When analyzed by subgroup, seroprotection rates were generally high in both study arms for all four serotypes, regardless of cancer type (S6 Table, S7A Fig.). When stratified by chemotherapeutic regimen, the seroprotection rates of serotypes 5 and 18C were lower in arm B in patients treated with fluoropyrimidine, although this did not reach statistical significance $(\mathrm{p}=0.312$ for serotype 5 and $\mathrm{p}=0.120$ for serotype 18C) (S8 Table, S7B Fig.).

The high seroprotection rate in our cohort was in part due to the high titers prior to vaccination for serotypes $6 \mathrm{~B}$ and 19A. However, the seroprotection rates in the two arms before vaccination did not differ significantly among the four serotypes (S9 Table, S10 Fig.), and the rates increased markedly for serotypes 5 and 18C (Fig. 2A, S5 Table), implying that immunogenicity in the two study arms might be similar.

\section{Immunogenicity assessed by seroconversion rate}

Another way to assess immunogenicity is to analyze the percentage of patients whose post-vaccination OPA titers increase greater than four-fold compared to the pre-vaccination level, which is otherwise known as the seroconversion rate $[14,15]$. The seroconversion rates varied according to 
serotype. For serotype 5, the rates were $>90 \%$ in both arms. However, for serotype $6 \mathrm{~B}$, the rates were $56.0 \%$ in arm $\mathrm{A}$ and $72.7 \%$ in arm B. Nevertheless, the rates did not differ significantly between the two arms (Fig. 2B, S11 Table).

When stratified by cancer type, the seroconversion rates in arms A and B were similar for both cancer types. In gastric cancer, arm $\mathrm{B}$ had higher rates than arm A for serotypes $6 \mathrm{~B}$, $18 \mathrm{C}$, and 19A, although these differences were not statistically significant (S12 Table, S13A Fig.). The results were similar when stratified by chemotherapeutic regimen. The seroconversion rates of all four serotypes were similar in the two study arms in patients treated with fluoropyrimidine. In the fluoropyrimidine-treated subgroup, the seroconversion rates were lower in arm B for serotypes $5(\mathrm{p}=0.120)$ and 19A $(\mathrm{p}=0.434)$, but this also did not reach statistical significance (S13B Fig., S14B Table).

\section{Subgroup analysis of patients with neutropenia or redu- ced-dose of chemotherapy}

Neutropenia is one of the most common adverse events of cytotoxic chemotherapy and it reflects the severity of bone marrow suppression. Therefore, we aimed to see whether the serologic responses to PCV13 were attenuated in patients whom neutropenia had occurred during the period of serum sampling. There were 20 patients who had neutropenia during the study period. Most of them were grade 1 or 2, and two patients had grade 3 neutropenia (based on Common Terminology Criteria for Adverse Events [CTCAE], ver. 5.0). We subclassified the study participants (control [n=67] vs. neutropenia $[n=20])$ and compared the immunogenicity assessments between the two groups. The GMT and foldincreases of titers, seroprotection, and seroconversion rates were all comparable between the two groups without statistical significance (S15-S18 Tables).

Chemotherapy doses are frequently modified based on the patients' general condition or tolerability. We also assessed whether reduced-dose of chemotherapy affects the serologic responses to vaccination. In our cohort, chemotherapy doses were reduced in 45 patients and all of them were reduced to $80 \%$ of the standard regimen during the study period. We again subclassified the patients into two groups (full-dose $[n=42]$ vs. reduced-dose $[n=45])$ and compared the immunogenicity parameters. No significant differences were seen in GMT and fold-increases, seroprotection, and seroconversion rates between the two groups (S19-S22 Tables).

\section{Subgroup analysis of patients treated with CAPOX}

In our dataset, CAPOX was the most commonly used regimen in both study arms. To analyze the immunogenicity in the background of a single regimen, we also compared the immunogenicity in patients who underwent chemotherapy with CAPOX for gastric or colorectal cancer. The geometric mean and fold increases, seroprotection, and seroconversion rates were mostly similar between the two groups (S23-S26 Tables). For geometric mean of titers, post-vaccination GMT for serotype 18C and the fold increase of GMT for serotype 6B were significantly higher in arm B (S23 and S24 Tables). Seroconversion rate of $6 \mathrm{~B}$ was also significantly higher in arm B. These results corroborate our key conclusion since vaccine administration seems to be at least non-inferior in the background of an identical chemotherapeutic regimen (S23-S26 Tables).

\section{Adverse events}

During the study, adverse events were reported on the day of vaccine administration, and 28-day post-vaccination. Six events were reported in am A and five were reported in arm $B$ which were all either grade 1 or 2 based on CTCAE ver. 5.0 (S27 Table). All events were reported on day 28 and no events were reported on the day of vaccine administration.

\section{Discussion}

This study demonstrated that administering PCV13 on the first day of adjuvant chemotherapy in patients with gastric or colorectal cancer is not inferior to administering the vaccine 2 weeks prior to chemotherapy. To the best of our knowledge, this is the first study to assess the immunogenicity of PCV13 in cancer patients undergoing systemic chemotherapy. Guidelines that recommend $\mathrm{a} \geq 2$-week interval between vaccination and chemotherapy stem mainly from limited studies performed with polysaccharide vaccines or PCV7 in patients with hematologic malignancies [16-18]. These studies found that better immunogenicity was acquired when vaccines were administered before initiation of chemotherapy. However, the optimal timing for PCV13 vaccination has not been properly studied in patients with solid tumors who are scheduled to receive repeated cycles of chemotherapy over a long period.

In this study, we assessed immunogenicity by analyzing the pre- and post-vaccination MOPA results from serum samples. Pre- and post-vaccination seroprotection and seroconversion rates in the two study arms were similar. However, these findings are inconsistent with pneumococcal vaccination studies performed for hematologic malignancies, which favored $\mathrm{a} \geq 2$-week interval between vaccine administration and initiation of chemotherapy $[17,18]$. One possible explanation for this discrepancy could be the difference in 
severity of chemotherapy-induced bone marrow suppression between the regimens used to target solid tumors and hematologic malignancies. While regimens for gastric or colon cancer do not usually cause severe bone marrow suppression, those targeting leukemia or lymphoma generally induce more severe cytopenia. This can also be inferred from a previous study that tested the serologic response to influenza vaccine in colon or breast cancer patients. Although immunogenicity to the vaccine did not differ significantly between the two populations (at 5 days or 16 days after chemotherapy), responses were generally lower in breast cancer patients who were treated with more cytotoxic regimens [5]. In our study, 20 patients had neutropenia during the assessment period, but their severities were low and did not show a significant difference in the serologic responses to PCV13.

This study has several limitations. First, we tested only four of the 13 serotypes covered by PCV13 using immunogenicity assays. Although our results consistently showed comparable immune responses for all four tested serotypes assessed using various methods, larger studies testing all serotypes are warranted. Additionally, this study included only patients undergoing adjuvant chemotherapy for gastric or colorectal cancer. It is unclear whether the results would be similar for other types of cancers treated with different chemotherapeutic regimens, or in more advanced stages requiring palliative therapies.

Despite these limitations, this study provides clear evidence that administering PCV13 on the first day of adjuvant chemotherapy is not inferior to vaccinating 2 weeks prior to starting chemotherapy in gastric and colorectal cancer patients. Further prospective trials with larger samples testing all serotypes covered with PCV13 are necessary to confirm whether this can be generalized to larger populations.

\section{Electronic Supplementary Material}

Supplementary materials are available at Cancer Research and Treatment website (https:// www.e-crt.org).

\section{Conflicts of Interest}

Conflict of interest relevant to this article was not reported.

\section{Acknowledgments}

We wish to thank all the participants of this study. We are grateful to Korean Cancer Study Group (KCSG), Jang HC and Oh IJ for the collaborative advice. We thank the Song JY's laboratory staff for the support during laboratory experiments.

\section{Author Details}

${ }^{1}$ Division of Hematology-Oncology, Department of Internal Medicine, Chonnam National University Hwasun Hospital, Chonnam National University College of Medicine, Hwasun, ${ }^{2}$ Department of Hematology/Oncology, School of Medicine, Kyungpook National University, Daegu, ${ }^{3}$ Division of Medical Oncology, Department of Internal Medicine, Yonsei University of College of Medicine, Seoul, ${ }^{4}$ Department of Preventive Medicine, Chonnam National University Medical School, Hwasun, Korea, ${ }^{5}$ Department of Biostatistics and Bioinformatics, Duke University, Durham, NC, USA, 'Division of Infectious Disease, Department of Internal Medicine, Korea University College of Medicine, Seoul, Korea

\section{References}

1. Rubin LG, Levin MJ, Ljungman P, Davies EG, Avery R, Tomblyn M, et al. 2013 IDSA clinical practice guideline for vaccination of the immunocompromised host. Clin Infect Dis. 2014;58:e44-100.

2. Shildt RA, Boyd JF, McCracken JD, Schiffman G, Giolma JP. Antibody response to pneumococcal vaccine in patients with solid tumors and lymphomas. Med Pediatr Oncol. 1983;11: 305-9.

3. Nordoy T, Aaberge IS, Husebekk A, Samdal HH, Steinert S, Melby $\mathrm{H}$, et al. Cancer patients undergoing chemotherapy show adequate serological response to vaccinations against influenza virus and Streptococcus pneumoniae. Med Oncol. 2002;19:71-8.

4. Hung TY, Kotecha RS, Blyth CC, Steed SK, Thornton RB, Ryan $\mathrm{AL}$, et al. Immunogenicity and safety of single-dose, 13-valent pneumococcal conjugate vaccine in pediatric and adolescent oncology patients. Cancer. 2017;123:4215-23.

5. Wumkes ML, van der Velden AM, Los M, Leys MB, Beeker A, Nijziel MR, et al. Serum antibody response to influenza virus vaccination during chemotherapy treatment in adult patients with solid tumours. Vaccine. 2013;31:6177-84.

6. Rodgers GL, Esposito S, Principi N, Gutierrez-Brito M, DiezDomingo J, Pollard AJ, et al. Immune response to 13-valent pneumococcal conjugate vaccine with a reduced dosing schedule. Vaccine. 2013;31:4765-74.

7. Moore MR, Link-Gelles R, Schaffner W, Lynfield R, Holtzman C, Harrison LH, et al. Effectiveness of 13-valent pneumococcal conjugate vaccine for prevention of invasive pneumococcal disease in children in the USA: a matched case-control study. Lancet Respir Med. 2016;4:399-406. 
8. Song JY, Nahm MH, Moseley MA. Clinical implications of pneumococcal serotypes: invasive disease potential, clinical presentations, and antibiotic resistance. J Korean Med Sci. 2013;28:4-15.

9. Song JY, Cheong HJ, Tsai TF, Chang HA, Choi MJ, Jeon JH, et al. Immunogenicity and safety of concomitant MF59-adjuvanted influenza vaccine and 23-valent pneumococcal polysaccharide vaccine administration in older adults. Vaccine. 2015;33:4647-52

10. Burton RL, Nahm MH. Development and validation of a fourfold multiplexed opsonization assay (MOPA4) for pneumococcal antibodies. Clin Vaccine Immunol. 2006;13:1004-9.

11. Beyer WE, Palache AM, Luchters G, Nauta J, Osterhaus AD. Seroprotection rate, mean fold increase, seroconversion rate: which parameter adequately expresses seroresponse to influenza vaccination? Virus Res. 2004;103:125-32.

12. Song JY, Moseley MA, Burton RL, Nahm MH. Pneumococcal vaccine and opsonic pneumococcal antibody. J Infect Chemother. 2013;19:412-25.

13. Romero-Steiner S, Musher DM, Cetron MS, Pais LB, Groover JE, Fiore AE, et al. Reduction in functional antibody activity against Streptococcus pneumoniae in vaccinated elderly indi- viduals highly correlates with decreased IgG antibody avidity. Clin Infect Dis. 1999;29:281-8.

14. Kumar D, Rotstein C, Miyata G, Arlen D, Humar A. Randomized, double-blind, controlled trial of pneumococcal vaccination in renal transplant recipients. J Infect Dis. 2003;187: 1639-45.

15. Durando P, Faust SN, Fletcher M, Krizova P, Torres A, Welte T. Experience with pneumococcal polysaccharide conjugate vaccine (conjugated to CRM197 carrier protein) in children and adults. Clin Microbiol Infect. 2013;19 Suppl 1:1-9.

16. Addiego JE Jr, Ammann AJ, Schiffman G, Baehner R, Higgins G, Hammond D. Response to pneumococcal polysaccharide vaccine in patients with untreated Hodgkin's disease: Children's Cancer Study Group Report. Lancet. 1980;2:450-2.

17. Frederiksen B, Specht L, Henrichsen J, Pedersen FK, PedersenBjergaard J. Antibody response to pneumococcal vaccine in patients with early stage Hodgkin's disease. Eur J Haematol. 1989;43:45-9.

18. Sinisalo M, Vilpo J, Itala M, Vakevainen M, Taurio J, Aittoniemi J. Antibody response to 7-valent conjugated pneumococcal vaccine in patients with chronic lymphocytic leukaemia. Vaccine. 2007;26:82-7. 